\title{
Quality of Life of Student Living with Early Decompressive Adrenal Fatigues in Pharmacy Institution in Pune, India
}

\author{
Rahul A Hajare* \\ Department of Biologics Development and Protein Chemistry, International Agency for Standards and Rating, India
}

*Corresponding author: Rahul A Hajare, Department of Biologics Development and Protein Chemistry, International Agency for Standards and Rating, Indian Council of Medical Researcher New Delhi, Researcher, National AIDS Research Institute Pune, India

Submission: 眥 May 16, 2018; Published: 笽June 08, 2018

\begin{abstract}
Early decompressive adrenal fatigues have one of the leading causes of neurological disorders and stroke in pharmaceutical Institutions. Health has the ability of a biological system convert into personal satisfaction. The World Health Organization (WHO) literature for human health in a broader sense in its 1948 constitution as "A state of complete physical and well-being and not merely the absence of disease or infirmity". It has been subject to controversy, in particular as poor transportation facility in pharmacy institution lacking operational value, the ambiguity in developing low health strategies and because of the problem created by use of the word "social determinants of health", which makes it practically impossible to achieve healthy environments. Understanding student health and disease with private based co-educational pharmaceutical institution has not transportation facility and low quality food in suburban areas of developing cities in India cannot ignore. Early decompressive adrenal insufficiency has accepted in medical science due to health imbalance. Adrenal fatigue especially from junior students to senior students living with private institution with poor quality of life and facility can translate into permanent disability in the world. We cannot compare adrenal fatigue with joint pain. Adrenal fatigue cannot ignore because it has weaken immunity has unfortunately not measured in the above series.
\end{abstract}

Keywords: Adrenal Fatigues; Digestive Problems; Fibromyalgia; Immunity; Life-Styles

\section{Introduction}

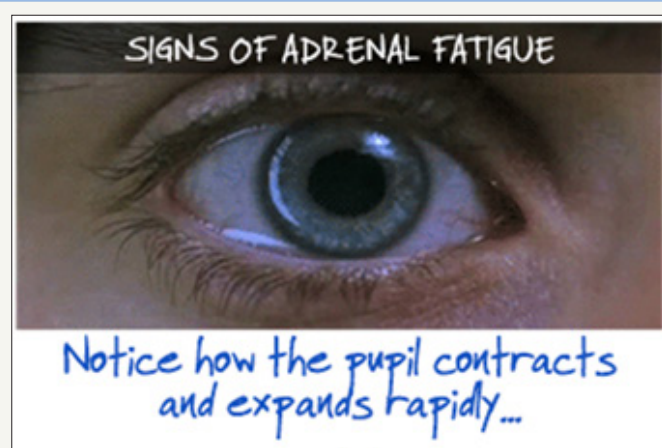

(a)

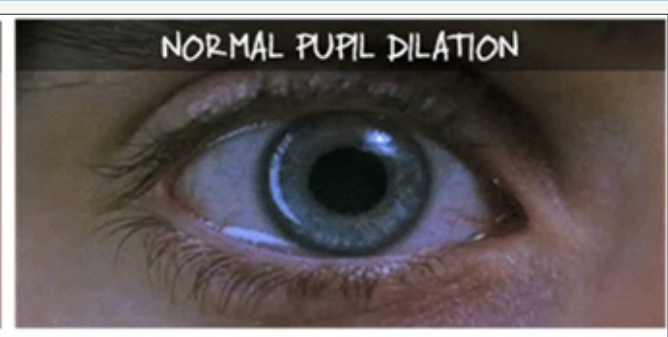

You DON'T HAVE Adrenal Fatigue

(b)

Figure 1: Cross examination of eye results indicate about the energy levels.

A Teacher has a person who teaches in school rather than, especially helps others to shows knowledge, competence and value, playing a role of mother [1]. The clinicians has faced with difficult decision of how to best treat these students as there are no evidence based guidelines regarding the management of such complications. The American Heart Association has suggested only empirical therapies to replacement this surgery has difficult for acceptance of low income country. Body mechanism has its own machinery to shape up body in symmetry [2,3]. Body shapes have been depends upon the immunity divided by twenty four hours. Meanwhile adrenal fatigue has collection of nonspecific symptoms, such as body aches, fatigue, nervousness, sleep disturbances, mouth odor, itching, and digestive problems (Figure $1 \& 2$ ). These symptoms often popular in poor transportation facility Institutions, but it has not an accepted medical diagnosis. It can be developed after a years and shows inches reduce the height of leg. Adrenal glands produce 
a variety of hormones that has essential to life. The medical adrenal insufficiency (Addison's disease) refers to inadequate production of one or more of these hormones as a result of an underlying disease. Behaviour and symptoms of adrenal insufficiency cannot ignore fatigue, body bend, and unseen weight loss, lightheadedness, loss of body hair, dark skin [4,5]. Adrenal insufficiency can be diagnosed by blood tests and special stimulation tests that show inadequate levels of adrenal hormones. The unproven theory behind adrenal fatigue has that your adrenal glands are unable to keep pace with the demands of perpetual fight-or-flight. As a result, they cannot produce quite enough of the hormones need to feel good. Existing blood tests, according to this theory, has not sensitive enough to detect such a small decline in adrenal function but body is. It is frustrating to have persistent symptoms physician cannot readily explain. But accepting a medically unrecognized diagnosis from an unqualified pharmaceutical practitioner could be worse. Unproven remedies for so-called adrenal fatigue may leave feeling sicker, low level of love hormone while the real cause such as depression or fibromyalgia, continues to take its toll.

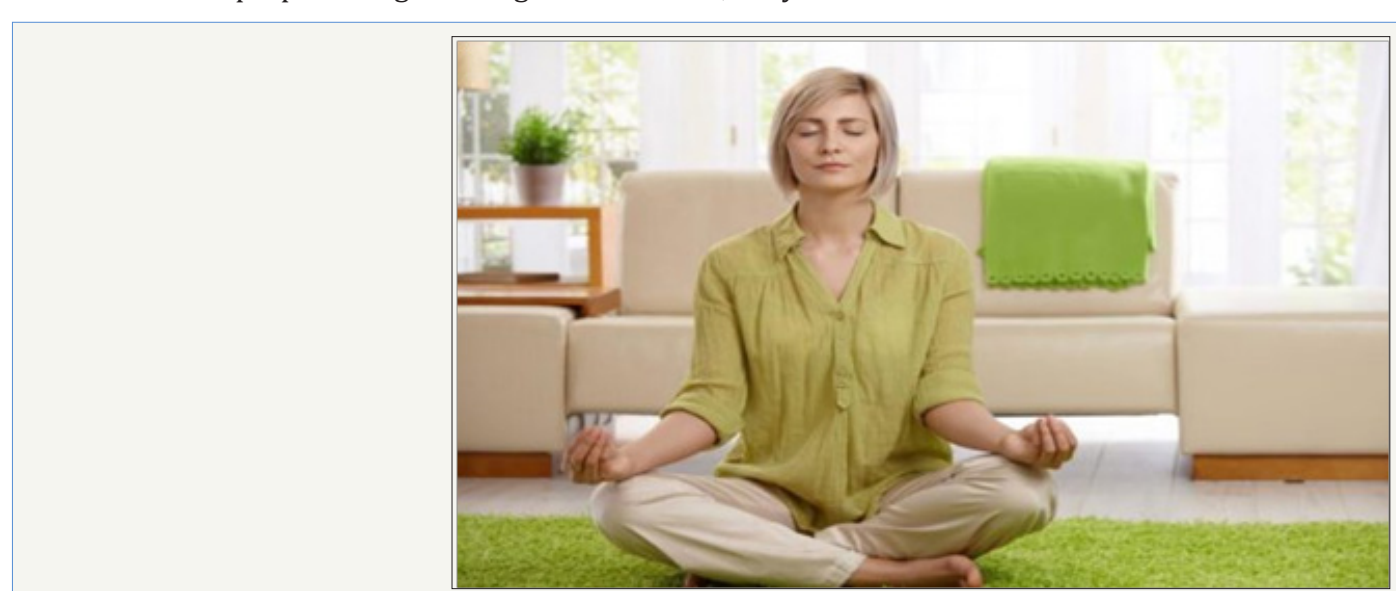

Figure 2: You Don't Have Adrenal Fatigue.

\section{Summary}

In simple terms, adrenal fatigue has not a genetic disorder associated with blood. It is caused by the lack of or errors in immunity in charge for production of hormone, a protein present in the red blood cells. "The complexity of the disease depends on the cross mutations involved in the life style, and their interplay. While adrenal fatigue seen a condition that affects educated pharmaceutical students of individuals across the serving himself with low sanitation and less transport facility, so they cannot produce love hormone enough its awareness is not given as much of importance as the other health conditions such as tounge cancer or diabetes. According to the World Health Organization, and other studies adrenal fatigue can destroy the immunity in individuals and Indian people suffering from the disease [6,7]. It is high time to spread awareness and take appropriate actions about this disease. Here are some facts that can know about this deadly condition. People with this disorder usually do not exhibit any symptoms, which makes it complex to identify. Healthy people can detect the adrenal fatigue. It is thus worthwhile to get tested, in case, any of students or junior students have some form of a disease. The other kind of adrenal fatigue is Alpha and Beta depends on the time of adrenal insufficient. In case of alpha adrenal fatigue, at least one of the alphas has an abnormality. In beta adrenal fatigue, the beta is affected. Each of the forms has sub-types, which vary in indications and rigorousness $[8,9]$. In case both the parents are carriers for adrenal fatigue, they will have 25 per cent risk to have a child affected with adrenal insufficiently. The India story, India, ranks on top of the list when it comes to adrenal fatigue.

\section{Symptoms}

Some of the common symptoms of the condition include fatigue, weakness, bone deformities, especially in the face, pale appearance or yellow skin, slow growth rates, lowered immunity levels, iron overload and heart diseases.

\section{How to prevent it}

Transportation is the movement of humans and goods from one location to other, including rowed and sailed vessels, dates back to time immemorial, and has the only efficient way to transport to reach in specific period of time and handling duty in time so It has physical and psychological effects, including influencing social behavior and emotion due to oxytocin is produced, a part of the brain.

\section{What students can do}

One should pursue universal immunization to prevent exposure from adrenal fatigue. It is recommended to consume food which has more in calcium and in proper time and less on iron as the students undergo frequent abdominal pain. People living with adrenal insufficiency can use cinnamon water. Sticking to a healthy diet and routine exercise helps to battle the disease. Campaign against no push more adrenal fatigues in private based co-educational pharmaceutical institution in India has been tested in academic year 2016-2018. The privately merge pharmaceutical institution co-educational controlled by management in regards to traditional laws of pharmacy council of India has been accepted, is the first of its substandard facility. Born out of the Indian legacy its mandate is to provide "high throughput, forensic quality, and metabolic health to support large scale pharmaceutical people as 
well as basic need with understanding and student stratification $[10,11]$. As global life-styles change we are seeing increasing cases of obesity, drowsiness, bending, leg bend, irritation, low metabolic rate, diabetes, skin pigmentation and mental health issues. This not only affects a student's quality of life but also places increased strain on the health-care systems to provide the right treatment whilst managing costs closely. Metabolic disorder offers a unvaluable and ununique insight into the underlying students of diseases as well as the students ' individual "behavioral", diet $[12,13]$, health status, age and stress. To deliver this information the analytical data generated in processed via a variety of analyzing different age group students and analysis methodologies to deliver the relevant biochemical information. These platforms employed vary from simple multi variant analysis to highly complex based analysis reported in earlier and has presented in a format ready for interpretation by medicine. Facility comprises of high field instruments, accurate result as well as dedicated training facility. In this presentation we will discuss the development of professional's platform students and students as well as a detailed discussion on the workflow, validation, reporting, explore of knowledge and decision making process. The full presentation will cover the development and validation of the "facilities' screening methods for transportation facility, cleanliness, separate dumping area of institutional scrap, bio safety institutional committee, Scientific Advisory Committee (SAC), additional other choice of accreditation and basic water drinking facility, profiling using junior student aptitude test / senior aptitude test adhere yearly, as well as describe the use of academic towards the society as an initial screen to eliminate contaminated unforensic procedures to harm the health related issues of students as well as to fulfill their acute shortage, to strengthen the educational health delivery system for safer place $[14,15]$. The quantitative targeted assays of pain management will also be discussed the various parameter such as student behavioral study, student reporting time, buffer time for students. University and educational inspectors may benefit from considering pharmacological approaches in the context of improving their university's attendance rates and academic performance. Presently it has one intervention and it will be updated regularly. There has been large number of other complaints that are associated with adrenal fatigue cannot avoided anxiety, asthma, allergies or respiratory complaints, Dark circles under the eyes, dizziness, depression, dry skin, extreme tiredness an hour after exercise, insomnia joint pain, lines in fingertips, loss of muscle tone, low blood pressure, lower back pain, high levels of fatigue each day after theory class, inability to handle stress, cravings for salty foods, higher energy levels in the evenings, weak immune system $[16,17]$. As we discuss in the adrenal fatigue solution, one of the major causes of adrenal fatigue has getting insufficient sleep. Early decompressive adrenal fatigues represent an aggressive life approach, especially for the students who develop opportunistic hemorrhagic complications. The decision to proceed with major surgical intervention requires a competent multi-disciplinary team as well as an open discussion with relative students as depressive adrenal fatigue may preserve both life and functional ability in well selected student. More research has needed in this field to elucidate the potential for both modalities in appropriate students. Getting more rest has therefore one of the best ways to recover. However, when suffering from adrenal fatigue many students wake up extremely tired and 'foggy', even after getting a long sleep. Zero aggression lifestyle and not eat food at any time in a day [18]. Selected food may get benefited for people living with adrenal insufficiency (Figure 2).

\section{Conclusion}

Student has report late to school and affects their learning frequently; tardiness is associated with lower grades and lower degree.

The author Dr Rahul Hajare has Fellow Indian Council of Medical Research New Delhi.

\section{Acknowledgment}

I expressed my deep gratitude to Renowned Laboratory Scientist, Retired Director of NARI Respected Dr. Ramesh S. Paranjape Immunologists. I express my sincere gratitude towards Respected Sir for motivation and being great knowledge source for this work

\section{References}

1. Rahul (2018) Instructional Teaching Strategy Can Boost an Activator of Students. J Yoga \& Physio 4(4): 555642.

2. Rahul Hajare (2018) The True Principal Health and Investigation of The High Burnout Stages Experienced by Professors Working in Pharmacy Institutions Pune University and Related Factors: an Important Study. Medical Research and Clinical Case Reports 1(2): 66-72.

3. Rahul H (2018) Regulation of Pharmacy Council of India and Assessment of Quality Life Among single Mother-By-Choice 'Residing in slums Linkage Pharmaceutical Institutions in Pune, India. Drug Des Int Prop Int J 1(3).

4. Rahul H (2018) A Short Review on "Social and Behavioural Research: Tool for Identify Alcohol Dependency Adult Men in Service Privately Managed Pharmaceutical Institutions in India". Trends Tech Sci Res 1(5): 555575

5. Rahul H (2018) Self-Explanatory Non-Funded Project Study of Dysmenorrhea and Impact on Quality of Life in Rajgad Dnyanpeeth's College of Pharmacy-Savitribai Phule Pune University Pune. Developments Clin Med Pathol 1(1).

6. Rahul H (2018) The Biosafety Against Privately Managed Pharmacy Institution in Savitribai Phule Pune University is Not Such a Simple Shot. Int J Nep \& Uro Dis 2(2): 06-07.

7. Rahul H (2017) Understanding Academic and Educational Problems Fit for Purpose in the Contributing to Attentional and Learning Difficulties in Our Children?. Glob J Oto 11(5): 555822.

8. Rahul H (2018) Why No More Apes Evolving Into Humans. Res Med Eng Sci 4(4).

9. Rahul H (2018) There is no Cure for the Cancer of Stupidity. Organic \& Medicinal Chem IJ 5(1):555655.

10. Rahul H (2018) Classical Approach to Management of Menopause: Extended Scientific Literature. Adv Res Gastroentero Hepatol 8(4): 555744

11. Rahul Hajare (2017) Live and Let Live: Acceptance of Learning Disability of People Living With Co-Educational Pharmaceutical Institute SelfFinanced and Privately Managed Remote Areas in India Where Stigma and Discrimination Persist. Current Opinions in Neurological Science 1(6): 311-313. 
12. Rahul H (2017) A Defense of Unqualified Pharmaceutical Confidentiality. Glob J Add \& Rehab Med 4(3): 555636.

13. Rahul H, Rajkumar Shete (2017) Process from Biosafety Working Style to Accreditation Trends Driving Self-financed Private Pharmacy Institution in Remote Areas Individuals in India. J Tradit Med Clin Natur:1(2)

14. Rahul Hajare (2018) Assessment of the Depression-Level Effectiveness of the Curse Words in Young Adults in Private Co-Educational Pharmaceutical Instituions in Pune University, India: A Preplanned, Causal-Pathway-Based Analysis. Curr Tr Gatsr \& Hepatol 1(1).

15. Rahul H (2018) Shocking Link Of Rapid Rise In Pre-Cancer Among The Healthy Adults In Pharmaceutical Institution Pune University India Living With Low Oxygen And Poor Sanitation Environment: Affordable Treatment Rate Three-Times Over. Glob J Add \& Rehab Med. 2018; 5(5): 555673.
16. Rahul H (2018) Two Trajectorieess a Promise of Reform and Ashaaram Pattern what are we Missing?. Orthop \& Spo Med Op Acc J; 1(2).

17. Rahul H (2017) Case-Study of Transference and Health Effects on the Senior Citizens who not having Children; Measures need to be put in Place to Fill the Care Gap in Pune Areas Suburban Hospitals and Nursing Homes: An Exploratory Report. MOJ Clin Med Case Rep 7(5): 00220.

18. Rahul H, Rajkumar Shete (2017) Process from Biosafety Working Style to Accreditation Trends Driving Self-financed Private Pharmacy Institution in Remote Areas Individuals in India. J Tradit Med Clin Natur: 1(2).

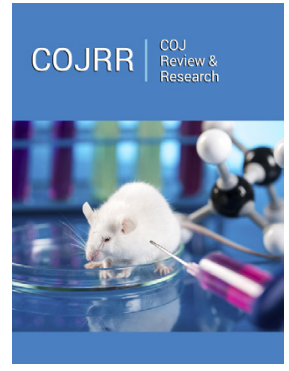

COJ Reviews \& Research

\section{Benefits of Publishing with us}

- High-level peer review and editorial services

- Freely accessible online immediately upon publication

- Authors retain the copyright to their work

- Licensing it under a Creative Commons license

- Visibility through different online platforms 